\title{
EL GENERO Aspergillus EN LA ATMOSFERA DE LA HABANA (CUBA)
}

\section{(The Aspergillus genus in the atmosphere of La Habana, Cuba)}

\author{
* Teresa I. Rojas; *Llanes, N.; *Benitez, M. \& \\ **Aira, M.J. y ***Malagón, $\mathbf{H}$. \\ *Departamento de Microbiología y Virología. Facultad de Biología. \\ Universidad de La Habana (Cuba) \\ **Departamento de Botánica, Facultad de Farmacia, \\ Universidad de Santiago (España) \\ *** Departamento de Agrometeorología, Instituto de Meteorología (Cuba)
}

Palabras clave: Esporas, atmósfera, Aspergillus, La Habana, Cuba.

Key words: Spores, atmosphere, Aspergillus, La Habana, Cuba.

\section{RESUMEN}

Se ha realizado un estudio de la contaminación fúngica ambiental en la atmósfera de la ciudad de La Habana (Cuba), durante el período de lluvias de los años 2001 y 2002, utilizando un método volumétrico viable, que ha permitido conocer la concentración de unidades formadoras de colonias ( $\mathrm{ufc} / \mathrm{m}^{3}$ ) totales y caracterizar los géneros y especies más abundantes, en especial del género Aspergillus. Durante el año 2001 las concentraciones más elevadas de hongos se obtuvieron en agosto y septiembre y las más bajas en julio, mientras que en el año 2002 destacan los niveles de septiembre y octubre frente a los escasos niveles del mes de agosto. Al analizar la densidad relativa de los géneros identificados se observa que Aspergillus es el género más abundante en la atmósfera seguido de Penicillium y Cladosporium. Las especies más abundantes en el año 2001 y relativamente constantes en el tiempo fueron $A$. japonicus, A. niger y A. flavus, mientras en el año 2002, los más abundantes fueron: A. japonicus, A. fumigatus $y$ A. niger, siendo A. niger el único constante en el tiempo.

\section{INTRODUCCION}

De todos los tipos de microorganismos presentes en la atmósfera, los hongos representan el grupo más numeroso por su gran abundancia en los diferentes hábitat, especialmente el suelo. El pequeño tamaño de sus elementos de propagación y su fácil dispersión ambiental,

Recibido el 19 de Diciembre 2006

Aceptado el 5 de Marzo 2007

\section{ABSTRACT}

A study on environmental fungal contamination in the atmosphere of the City of La Habana (Cuba), during the rainy period in 2001 and 2002 was carried out. A viable volumetric method was used, making it possible to ascertain the concentration of units comprising total colonies $\left(\mathrm{ufc} / \mathrm{m}^{3}\right)$, and to characterise the most abundant genera and species, especially Aspergillus. Throughout 2001, the highest concentrations of fungi were collected in August and September, and the lowest in July; while in 2002, the highest levels were in September and October, compared to the minimal levels for the month of August. On analysing the relative density of the genera identified, it was found that the most abundant genus in the atmosphere was Aspergillus, followed by Penicillium and Cladosporium. The most abundant species and relatively constant over time in 2001 were A. japonicus, A. niger and A. flavus while in 2002 A. japonicus, A. fumigatus and A. niger; this specie was the only constant in time.

favorecen la presencia de altos niveles de sus esporas en la atmósfera (Rojas \& Martínez, 2000).

Las esporas fúngicas alcanzan concentraciones muy significativas en determinadas épocas del año y son las responsables del elevado porcentaje de pacientes sensibilizados a estos aeroalergenos y diagnosticados con problemas de alergia (Eggleston et al., 1998; Hasnain et al., 1998; Mediavilla et al., 1998). En la literatura se reporta que entre los hongos conocidos como alergénicos destacan los géneros Aspergillus, Penicillium, Cladosporium y Alternaria (Andersen, 1985) y las respuestas 
alérgicas son generalmente debido más bien a la inhalación de sus esporas que al material derivado del micelio (Dopazo et al., 2001). Esta inhalación puede desencadenar una variedad de síntomas respiratorios, como rinitis alérgica, asma, bronquitis crónica, etc., que dependen de la especie, de las condiciones, tanto del medio en el que se desarrolla el hongo como climáticas, y de la reactividad inmunológica del sujeto.

Son numerosos los trabajos realizados en todo el mundo sobre aeromicología que pretenden conocer el espectro de esporas fúngicas de una localidad, su variación en el tiempo y como pueden influir determinadas condiciones ambientales en su liberación y dispersión (Halwagy, 1994; Mitakakis \& Guest, 2001; La-Serna, Dopazo \& Aira, 2002; Sabariego, 2003), sin embargo, en Cuba, el recuento e identificación de esporas en ambientes exteriores ha sido poco estudiado (Arnold et al., 1987; Rojas et al., 2003, Llanes et al., 2004), a pesar de que las condiciones climatológicas de países tropicales, especialmente alta humedad y temperatura, son idóneas para el desarrollo de una gran variedad de microorganismos específicamente de hongos. De ahí la importancia de establecer una metodología para este tipo de estudio y demostrar la presencia de estos microorganismos en nuestro medio ambiente.

Este trabajo tiene como objetivos, realizar el monitoreo del aire exterior de la Ciudad de La Habana con el fin de determinar la carga viable fúngica $\left(u f c . \mathrm{m}^{-3}\right.$ de aire) diurna y estacional en la atmósfera durante el período de lluvia de los años 2001 y 2002, aislar, caracterizar e identificar los géneros presentes en el aire, en especial las especies del género Aspergillus, relacionando la carga viable y tipo de espora con los parámetros climatológicos (temperatura, humedad relativa, velocidad del viento).

\section{MATERIALES Y METODOS}

El muestreo se realizó en la azotea de la Facultad de Biología de la Universidad, situada a una altura de 35 metros del suelo, con un biocolector volumétrico viable (Aeroscopio Chirana), incorporando en la parte superior del mismo una placa con agar Sabouraud $+7.5 \%$ de $\mathrm{NaCl}$ a pH 5 (Rojas y Martínez, 2000). Se siguió un diseño diagonal en tres puntos de la azotea con una réplica de tres placas por punto y cada muestreo tuvo una duración de 1 minuto.

Se realizaron 12 muestreos con frecuencia mensual, entre mayo y octubre de los años 2001 y 2002.

Todas las placas Petri fueron incubadas en la oscuridad de forma invertida a $28^{\circ} \mathrm{C}$ durante 4-6 días. Transcurrido ese tiempo se realizó el recuento de las colonias emergentes en el medio de cultivo y se determinaron las ufc $/ \mathrm{m}^{3}$ de aire, teniendo en cuenta el coeficiente de cálculo según NRP201 (1987). Los integrantes del género Aspergillus fueron estudiados en los medios selectivos de agar malta y Czapeck siguiendo la metodología de Al-Musallam, 1980 y Klich, 2002.

Estudio intradiurno para determinar la mejor hora de muestreo. Con el fin de determinar la hora del día de mayor concentración de esporas, se realizaron tres muestreos con frecuencia horaria, desde las 8:00 hasta las 17:00, (3 placas/hora). Se obtuvieron 30 placas de cultivo por muestreo (con sus respectivas réplicas), en cada una de ellas se contabilizó el número de unidades formadoras de colonias por metro cúbico de aire, tanto las totales como las correspondientes a Aspergillus.

Los registros de humedad relativa (H.R.), temperaturas y velocidad del viento (VV), fueron suministrados por la estación meteorológica de Casa Blanca, perteneciente al Instituto de Meteorología. La densidad relativa de los géneros de hongos detectados se calculó según Smith (1980) y la relación existente entre el número de asilamientos de los hongos más abundantes y los parámetros meteorológicos fueron analizadas con el paquete estadístico TonyStat, aplicando el coeficiente de correlación por rangos de Spearman.

\section{RESULTADOS Y DISCUSION}

La concentración total de esporas en el aire varía a lo largo del día recogiéndose las concentraciones más elevadas en horas de la mañana (9:00-10:00) y las más bajas durante la tarde. Lo mismo ocurre al analizar el comportamiento de las esporas de Aspergillus cuyos máximos se localizan siempre antes del mediodía, por lo que se acuerda fijar la hora de los muestreos del resto del estudio a las 11:00 AM (Figura 1). Resultados similares han sido señalados por Rosas et al. (1992), en la ciudad de México, donde las esporas de dicho género se concentran mayoritariamente en horas tempranas de la mañana.

Según Lacey (1981), en la mayoría de los hongos anamórficos, los niveles de concentración de esporas en el aire se relacionan con las condiciones necesarias para su liberación (turbulencias, velocidad del viento, difusión del calor por circulación e inversiones de temperaturas). En nuestros resultados se corrobora que los picos de máxima concentración de hongos coinciden con los mínimos de velocidad del viento, no encontrándose una relación directa con la temperatura ni la humedad relativa dada la homogeneidad de sus valores.

Muestreos mensuales: En el año 2001 las concentraciones más elevadas de hongos se recogieron 


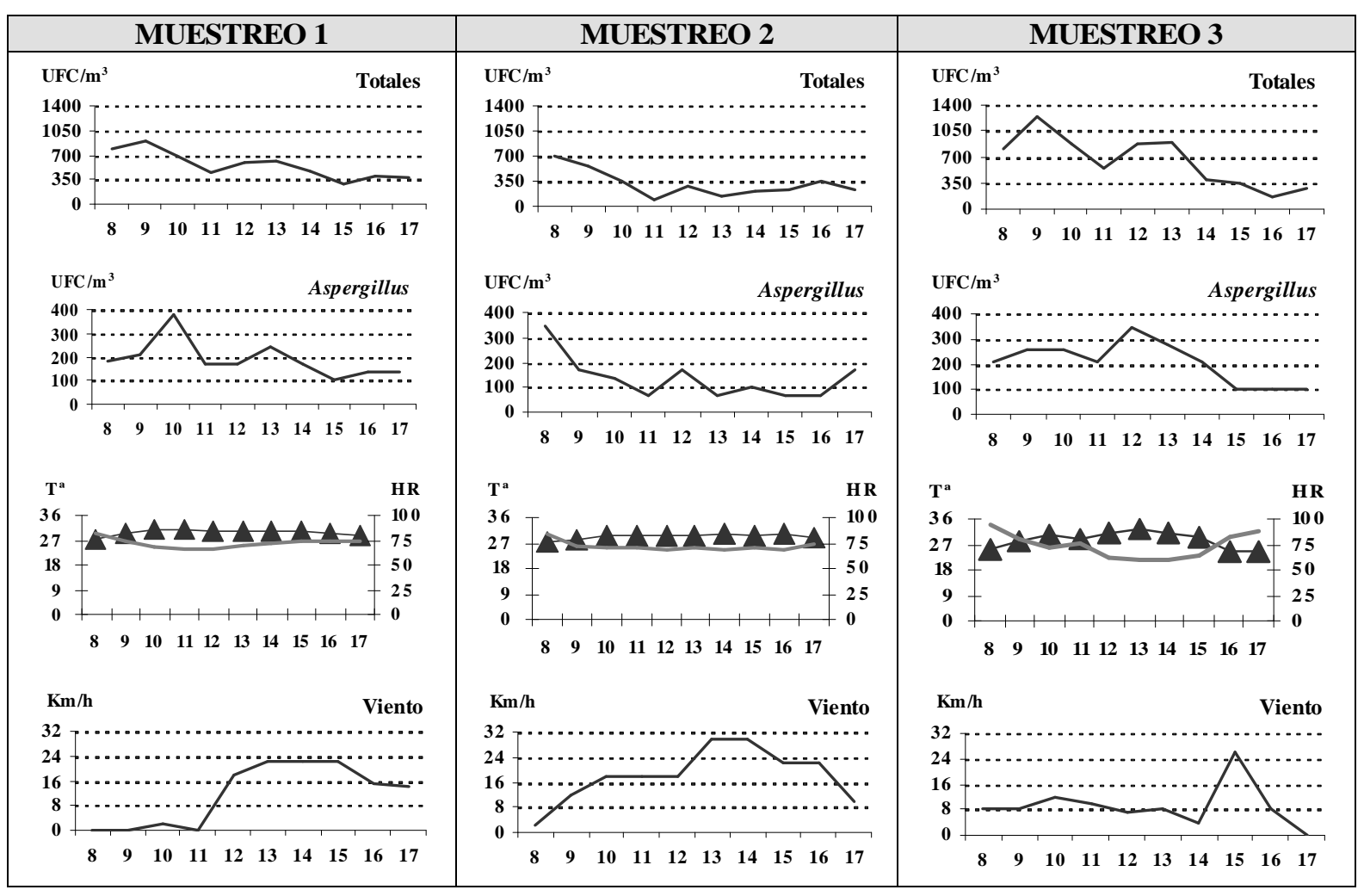

$-\mathrm{T}^{\mathrm{a}} \% \mathrm{HR}$

Figura 1. Variación intradiurna de la concentración de UFC.m³ ${ }^{3}$-totales y Aspergillus- y de los parámetros meteorológicos durante tres muestreos.

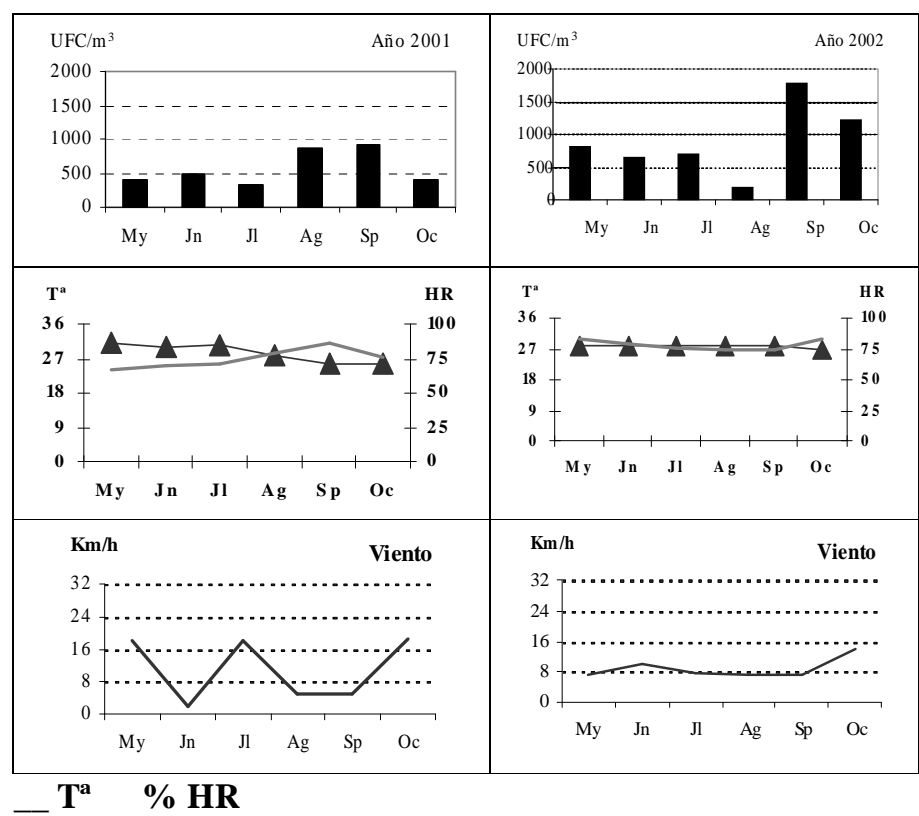

Figura 2.- Variación mensual de la concentración de hongos y de los parámetros meteorológicos en los dos años de muestreo

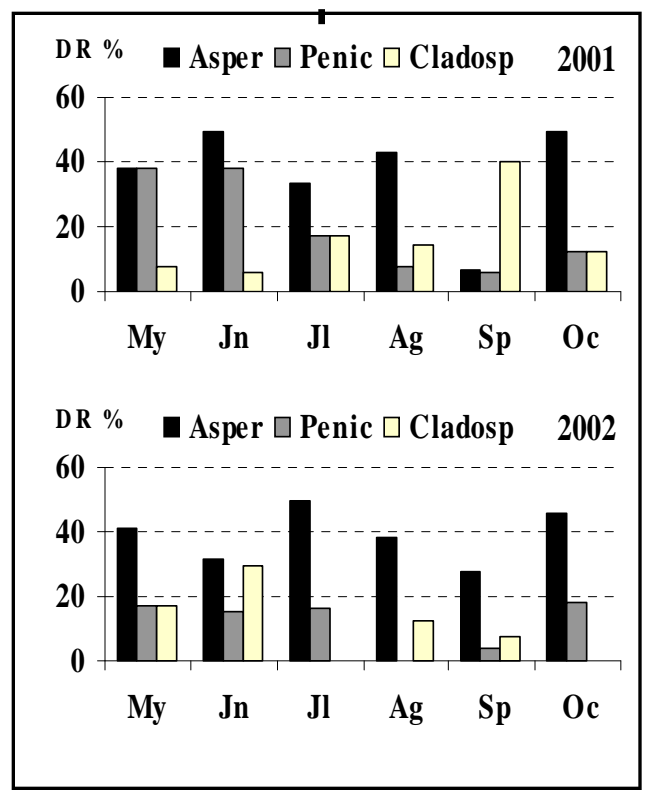

Figura 3.- Variación mensual de la concentración de Aspergillus, Penicillium y Cladosporium 
Tabla 1.- Correlación entre el número de aislamientos y los parámetros meteorológicos

\begin{tabular}{|l|c|c|c|c|c|c|}
\hline \multirow{2}{*}{$\begin{array}{c}* \mathrm{p}<0.05 \\
\text { n.s: No significativo }\end{array}$} & \multicolumn{2}{|c|}{ Aspergillus } & \multicolumn{2}{c|}{ Penicillium } & \multicolumn{2}{c|}{ Cladosporium } \\
\cline { 2 - 7 } & 2001 & 2002 & 2001 & 2002 & 2001 & 2002 \\
\hline HR (\%) & $-0.4857 \mathrm{n} . \mathrm{s}$ & $0.0000 \mathrm{n} . \mathrm{s}$ & $-0.2070 \mathrm{n} . \mathrm{s}$ & $0.7775^{*}$ & $0.6761 \mathrm{~ns}$ & $-0.1765 \mathrm{~ns}$ \\
\hline $\mathrm{T}^{\mathrm{a}}\left({ }^{\circ} \mathrm{C}\right)$ & $0.4286 \mathrm{n} . \mathrm{s}$ & $-0.1343 \mathrm{n} . \mathrm{s}$ & $0.2070 \mathrm{n} . \mathrm{s}$ & $-0.8232^{*}$ & $-0.5071 \mathrm{~ns}$ & $0.1343 \mathrm{~ns}$ \\
\hline Viento $(\mathrm{Km} / \mathrm{h})$ & $-0.6000 \mathrm{n} . \mathrm{s}$ & $-0.4414 \mathrm{n} . \mathrm{s}$ & $-0.4140 \mathrm{n} . \mathrm{s}$ & $0.5409 \mathrm{n} . \mathrm{s}$ & $-0.3719 \mathrm{~ns}$ & $-0.3531 \mathrm{~ns}$ \\
\hline
\end{tabular}

en agosto y septiembre y las más bajas en julio, mientras que en el año 2002 destacan los niveles de septiembre y octubre frente a los escasos niveles del mes de agosto (Figura 2). Estas diferencias pueden relacionarse con las condiciones climáticas, ya que aunque la temperatura y la humedad no sufrieron grandes variaciones, los meses de septiembre y octubre de 2001 fueron más lluviosos que los del 2002 (3,2 y 6,7 mm de precipitación en 2001 frente a 0 y $3,5 \mathrm{~mm}$ de precipitación en 2002 respectivamente en dichos meses). Es probable que también la velocidad y la dirección del viento ejerciera una cierta influencia, ya que los meses de mayor concentración de hongos en ambos años coinciden con velocidades bajas y dirección NNE.

Al analizar la densidad relativa (DR \%) de los géneros identificados en los diferentes meses se observa que Aspergillus es el género más abundante en la atmósfera seguido de Penicillium y Cladosporium (Figura 3).
Aspergillus está representado en los doce muestreos con una densidad relativa media entre el 37$39 \%$ en cada año; Penicillium aparece en once muestreos con una densidad media de $20-12 \%$ y finalmente Cladosporium se representa en nueve muestreos con una densidad media entre 16-11\% (Tabla 2).

$\mathrm{Si}$ analizamos estos resultados en relación con los parámetros meteorológicos que se han considerado, se observa que los picos máximos de Aspergillus en ambos años coinciden con los niveles mas bajos de humedad relativa (70-75\%) mientras que para Cladosporium los niveles de humedad son superiores (90\% en el 2001 y $80 \%$ en el 2002), lo cuál refleja que las esporas de Aspergillus se dispersan más fácilmente en ambiente seco mientras las de Cladosporium requieren más humedad.

Aplicando el test de Spearman se concluye que el número de aislamientos de los géneros Aspergillus y

Tabla 2.- Frecuencia de presencia mensual de todos los géneros y otras categorías durante los años 2001 y 2002

\begin{tabular}{|c|c|c|c|c|c|c|c|c|c|c|c|c|}
\hline \multirow[b]{2}{*}{$\begin{array}{l}\text { Géneros y otras } \\
\text { categorías fúngicas }\end{array}$} & \multicolumn{2}{|c|}{ Mayo } & \multicolumn{2}{|c|}{ Junio } & \multicolumn{2}{|c|}{ Julio } & \multicolumn{2}{|c|}{ Agosto } & \multicolumn{2}{|c|}{ Septiembre } & \multicolumn{2}{|c|}{ Octubre } \\
\hline & 2001 & 2002 & 2001 & 2002 & 2001 & 2002 & 2001 & 2002 & 2001 & 2002 & 2001 & 2002 \\
\hline Alternaria & & 17 & & & & & 7 & & & & & \\
\hline Aspergillus & 38 & 41 & 50 & 31 & 33 & 50 & 43 & 38 & 7 & 28 & 50 & 46 \\
\hline Aureobasidium & & & & 8 & & & & & & & & \\
\hline Chrysonilia & & & & & & & 14 & & & & & 9 \\
\hline Cladosporium & 8 & 17 & 6 & 30 & 17 & & 14 & 12 & 40 & 8 & 13 & \\
\hline Curvularia & & & & & & & & & & 4 & & \\
\hline Dematiaceae s/c & & 8 & & & & & & & & & & 9 \\
\hline Fusarium & & & & 16 & & & & & 27 & 24 & & \\
\hline Geotrichum & & & & & & & & & & 8 & & \\
\hline Levaduras & 8 & & & & 16 & & & & 7 & & & \\
\hline $\begin{array}{l}\text { Mitospóricos } \\
\text { hialinos s/c }\end{array}$ & & & & & & 25 & & 25 & & 24 & & 9 \\
\hline Penicillium & 38 & 17 & 38 & 15 & 17 & 17 & 8 & & 6 & 4 & 12 & 18 \\
\hline Rhizopus & & & & & & & & 25 & & & 25 & \\
\hline Syncephalastrum & 8 & & 6 & & 17 & & & & & & & \\
\hline $\begin{array}{l}\text { Trichoderma } \\
\text { Ulocladium }\end{array}$ & & & & & & 8 & 14 & & 13 & & & 9 \\
\hline
\end{tabular}



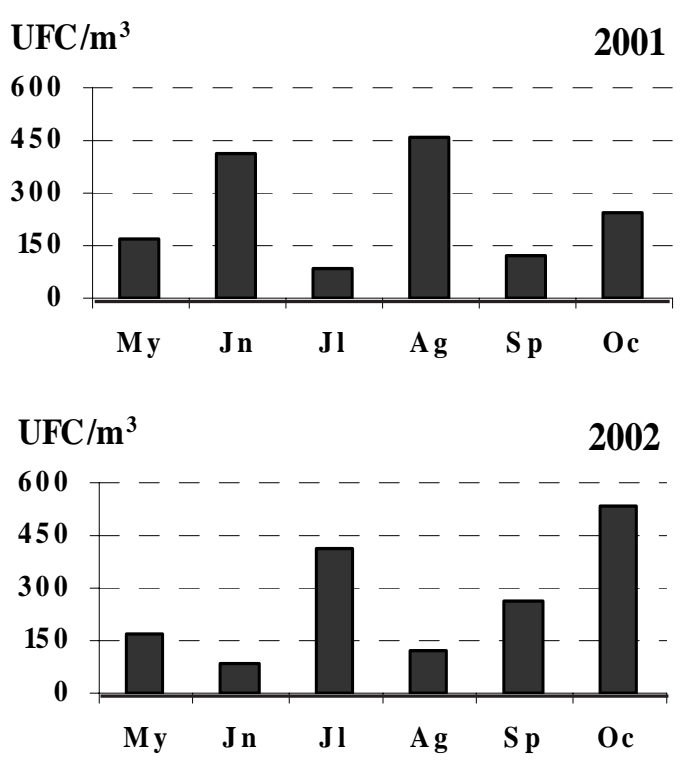

Figura 4.- Variación mensual de los integrantes del género Aspergillus en los dos años de muestreo

Cladosporium no muestran una correlación significativa desde el punto de vista estadístico con ninguno de los parámetros meteorológicos, sin embargo, para Penicillium la humedad relativa influye positivamente y la temperatura influye de forma negativa, explicando en ambos casos un elevado porcentaje de la variabilidad de los datos con un nivel de confianza del 95\% (Tabla 1).

Otros grupos de hongos tales como las levaduras y hongos mitosporicos hialinos (sobre todo Trichoderma) muestran una leve representatividad en el año 2001, mientras que en el año 2002 son más abundantes algunos Dematiaceos como Alternaria y mitospóricos hialinos como Fusarium (Tabla 2).

Al analizar el comportamiento de Aspergillus en ambos años, se observa que los meses de máxima concentración son junio y agosto en 2001 y julio y octubre en 2002 (Figura 4). Los resultados promedio de ambos años señalan una concentración máxima en el mes de junio $\left(491 \mathrm{ufc} / \mathrm{m}^{3}\right)$ y de octubre $\left(393 \mathrm{ufc} / \mathrm{m}^{3}\right)$. Las especies de Aspergillus identificadas en ambos años han sido $\boldsymbol{A}$. fumigatus Fresenius, $\boldsymbol{A}$. niger van Tieghem var. niger, $\boldsymbol{A}$. flavus Link., A. ornatus (Grupo) y A. japonicus Saito (Tabla 3). En el año 2001 la especie más abundante ha sido A. japonicus, con dos picos máximos en julio y agosto y con menor importancia destacaron A. niger var. niger y A. flavus, ambos con valores elevados en el mes de octubre. En el año 2002 los niveles más elevados correspondieron A. fumigatus y A. japonicus con máximos en julio y octubre respectivamente. Otras especies identificadas no comunes en el período de
Tabla 3.- Variación mensual de las especies de Aspergillus

\begin{tabular}{|l|c|c|c|c|c|c|c|}
\hline \multicolumn{1}{|c|}{ Taxa } & My & Jn & Jl & Ag & Sp & Oc & Total \\
\cline { 2 - 9 } A. fumigatus & & 41 & & & & & 41 \\
\hline A. niger & $\mathbf{4 1}$ & & & & $\mathbf{8 3}$ & $\mathbf{1 2 4}$ & $\mathbf{2 4 8}$ \\
\hline A. flavus & & $\mathbf{8 3}$ & $\mathbf{4 1}$ & $\mathbf{4 1}$ & & $\mathbf{1 2 4}$ & $\mathbf{2 8 9}$ \\
\hline A. ornatus & & & & 41 & & & 41 \\
\hline A. japonicus & $\mathbf{8 3}$ & $\mathbf{2 4 3}$ & $\mathbf{4 1}$ & $\mathbf{3 3 1}$ & & & $\mathbf{6 9 8}$ \\
\hline A. ochraceus & & 41 & & 41 & & & 82 \\
\hline Otros Asperg. & 41 & & & & 41 & & 82 \\
\hline & & $\mathbf{A n ̃ o}$ & $\mathbf{2 0 0 2}$ & & & \\
\hline A. fumigatus & & & $\mathbf{3 3 1}$ & & & & $\mathbf{3 3 1}$ \\
\hline A. niger & & $\mathbf{8 3}$ & $\mathbf{4 1}$ & $\mathbf{4 1}$ & $\mathbf{4 1}$ & $\mathbf{4 1}$ & $\mathbf{2 4 7}$ \\
\hline A. flavus & 165 & & & & & & 165 \\
\hline A. ornatus & & & & & 41 & & 41 \\
\hline A. japonicus & & & & & $\mathbf{1 3 8}$ & $\mathbf{3 3 1}$ & $\mathbf{4 6 9}$ \\
\hline A. tamarii & & & 41 & & & 41 & 82 \\
\hline Otros Asperg. & & & & 83 & 41 & 124 & 248 \\
\hline
\end{tabular}

muestreo, han sido A. ochraceus Wilhem concretamente en los meses de junio y agosto del año 2001 y A. tamarii Kita en julio y octubre del año 2002 (Tabla 3).

Como conclusiones de este estudio se puede indicar que los niveles de hongos en el aire exterior de la ciudad de La Habana fueron superiores en el año 2002 frente al 2001 con valores promedios anuales de $897 \mathrm{ufc} / \mathrm{m}^{3}$ frente a $575 \mathrm{ufc} / \mathrm{m}^{3}$. El análisis mensual también es superior en el año 2002 con la excepción del mes de agosto (869 ufc/ $\mathrm{m}^{3}$ en 2001 frente a $200 \mathrm{ufc} / \mathrm{m}^{3}$ en 2002). En cualquier caso los niveles son suficientemente elevados para justificar la continuación de estos estudios preliminares, con el fin de obtener una mayor información sobre la calidad del aire.

Dado que el género Aspergillus ha sido identificado con elevados niveles en el ambiente, también será necesario continuar con el estudio de caracterización de las especies representadas dado que sus esporas tienen una reconocida implicación con diversas enfermedades respiratorias de tipo alérgico e invasivas.

\section{REFERENCIAS}

AL_Musallam, A. (1980). Revision of the black Aspergillus species. Ph.D. Thesis. Utrecht, Netherlands: University of Utrecht.

Andersen, A. (1985). Microfungi in best and their relation to house dust mites. Grana 24:55-59

Arnold, G.R.W., Guerra , A., Rodríguez , N. (1987). Presencia de hongos en el aire del INIFAT. Reporte de Investigación del Instituto 
Instituto de Investigaciones Fundamentales en Agricultura Tropical, $\mathrm{N}^{\circ} .43,7$ pp. INIFAT.

Dopazo, A.; Hervés, M. \& Aira, M.J. (2001). Concentración de esporas de Alternaria, Cladosporium y Fusarium en la atmósfera de Santiago de Compostela (1996). Botánica Complutenses 25:83-91

Eggleston, P.A.; Rosenstreich, D.; Slavin, R. \& Malveaux, F. (1998). Relationship of indoor allergen exposure to skin test sensitivity in inner city asthma. J. Allergy Clin. Immunol. 102:563-570

Halwagy, M.H. (1994). Fungal airspora of Kuwait City, Kuwait, 19751987. Grana 33:340-345

Hasnain, S.M.; Al-Frayh, A.; Gad-El-Rab, M.O. \& Al-Sedairy, S. (1998). Airborne Alternaria spores: potencial allergic sensitizers in Saudi Arabia. Ann. Saudi. Med. 18: 497-501

Klich, M. A. (2002). Identification of common Aspergillus species. CBS, Utrecht, The Netherlands.

Lacey, J. (1981). The Aerobiology of Conidial Fungi. En: Biology of Conidial Fungi 1:373-416. Academic Press, London.

La-Serna, I.; Dopazo, A. \& Aira, M.J. (2002). Airborne fungal spores in the Campus of Anchieta (La Laguna, Tenerife/Carary Is.). Grana 41:119-123

Llanes, N.; Rojas,T.I.; Benítez, M.; Barrios, L.M.;Malagón, H. (2004). Hongos fitopatógenos detectados en el aire. Memorias del XI Congreso Científico del Instituto Nacional de Ciencias Agricolas (La Habana).

Mediavilla, A.; Angulo, J.; Infante, F.; Comtois, P.; Domínguez, E. (1998). Preliminary statistical modeling of the presence of two conidial types de Cladosporium in the atmosphere of Cordoba, Spain. Aerobiología 14:229-234
Mitakakis, T.Z. \& Guest, D.I. (2001). A fungal spore calendar for the atmosphere of Melbourne, Australia, for the year 1993. Aerobiología $17: 171-176$

NRP201. (1987). Análisis higiénico sanitario y ambiental. Métodos de ensayos microbiológicos, $6 \mathrm{pp}$.

Rojas, T.I.; Benítez, M.; Llanes, N.; Alba, L.; Aira, M.J.; Malagón, H. (2003). Género Aspergillus en la atmósfera de la Ciudad de La Habana. VII Simposio de Botánica, Ciudad de La Habana 24-28 de junio.

Rojas, T.I. \& Martínez, E. (2000). Monitoreo microbiano del aire: Criterios metodológicos. Contribución a la educación y protección ambiental. VI Taller de la Cátedra de Medio Ambiente. Vol. 1:110-115

Smith, G. (1980). Ecology and Field Biology. Harper \& Row, New York, $835 \mathrm{pp}$.

Sabariego, S.; Díaz de la Guardia, C. \& Alba, F. (2004). Incidencia de esporas de Alternaria y Cladosporium en la atmósfera de la ciudad de Almería (SE de España): relación con los parámetros meteorológicos. R.I.A.M

Rosas, I.; Calderón, C.; Escamilla, B. \& Ulloa, M. (1992). Seasonal distribution of Aspergillus in the air of an urban area: Mexico City. Grana 31:315-319 